\title{
Regulation and function of the atypical cadherin FAT1 in hepatocellular carcinoma
}

\author{
Daniela Valletta, Barbara Czech, Thilo Spruss ${ }^{1}$, \\ Kristian Ikenberg ${ }^{2}$, Peter Wild ${ }^{2}$, Arndt Hartmann ${ }^{3}$, \\ Thomas S.Weiss ${ }^{4}$, Peter J.Oefner ${ }^{5}$, Martina Müller, \\ Anja-Katrin Bosserhoff ${ }^{6}$ and Claus Hellerbrand*
}

Department of Internal Medicine I, University Hospital Regensburg, 93053 Regensburg, Germany, ${ }^{1}$ Institute of Pharmacy, University of Regensburg, 93053 Regensburg, Germany, ${ }^{2}$ Institute of Surgical Pathology, University Hospital Zurich, 8091 Zurich, Switzerland, ${ }^{3}$ Institute of Pathology, University Hospital Erlangen, 91054 Erlangen, Germany, ${ }^{4}$ Department of Pediatrics and Juvenile Medicine, University Hospital Regensburg, 93053 Regensburg, Germany and ${ }^{5}$ Institute of Functional Genomics and ${ }^{6}$ Institute of Pathology, University of Regensburg, 93053 Regensburg, Germany

*To whom correspondence should be addressed. Tel: +49 941944 7155; Fax: +49 9419447154

Email: claus.hellerbrand@ukr.de

In human cancers, giant cadherin FAT1 may function both, as an oncogene and a tumor suppressor. Here, we investigated the expression and function of FAT1 in hepatocellular carcinoma (HCC). FAT1 expression was increased in human HCC cell lines and tissues compared with primary human hepatocytes and non-tumorous liver tissue as assessed by quantitative PCR and western blot analysis. Combined immunohistochemical and tissue microarray analysis showed a significant correlation of FAT1 expression with tumor stage and proliferation. Suppression of FAT1 expression by short hairpin RNA impaired proliferation and migration as well as apoptosis resistance of $\mathrm{HCC}$ cells in vitro. In nude mice, tumors formed by FAT1-suppressed HCC cells showed a delayed onset and more apoptosis compared with tumors of control cells. Both hepatocyte growth factor and hypoxia-mediated hypoxia-inducible factor 1 alpha activation were identified as strong inducers of FAT1 in HCC. Moreover, demethylating agents induced FAT1 expression in HCC cells. Hypoxia lead to reduced levels of the methyl group donor $S$-adenosyl-L-methionine (SAM) and hypoxia-induced FAT1 expression was inhibited by SAM supplementation in HCC cells. Together, these findings indicate that FAT1 expression in $\mathrm{HCC}$ is regulated via promotor methylation. FAT1 appears as relevant mediator of hypoxia and growth receptor signaling to critical tumorigenic pathways in HCC. This knowledge may facilitate the rational design of novel therapeutics against this highly aggressive malignancy.

\section{Introduction}

Cadherins are major contributors to cell-cell adhesion in epithelial tissues and play a critical role in morphogenetic and differentiation processes during development, and in maintaining integrity and homeostasis in adult tissues (1). Several cadherins have been identified as potential tumor suppressors or oncogenic proteins depending on the type of cadherin and the type of cancer (2). The cadherin subfamily FAT is characterized by large extracellular domains containing 34 cadherin motifs, making it the largest of all cadherin molecules (3). The first identified member of this family, Drosophila Fat, has been shown to be involved in cell proliferation and survival as well as cellular polarity (4). FAT1 was identified as the first Fat-like protein in vertebrates (5). Mice lacking FAT1 exhibit perinatal lethality with defects in kidney and brain (6). Transcript variants derived from alternative

Abbreviations: CM, conditioned medium; DP, dipyridyl; HCC, hepatocellular carcinoma; HGF, hepatocyte growth factor; $\mathrm{HIF} 1 \alpha$, hypoxia-inducible factor 1 alpha; HSC, hepatic stellate cell; MAZ, Myc-associated zinc finger protein; mRNA, messenger RNA; PHH, primary human hepatocytes; SAM, $S$-adenosyl-1-methionine; shRNA, short hairpin RNA; siRNA, small interfering RNA; TMA, tissue microarray; VEGF, vascular endothelial growth factor. splicing exist, but their differential function just begins to be elucidated (7). Moreover, FAT1's mechanisms of action in normal and diseased tissues are incompletely understood $(3,8)$. In cancer, FAT1 appears to act as tumor suppressive or oncogenic in a context-dependent manner (8). Frequent loss of heterozygosity of FAT1 has been described in oral squamous cell carcinomas and astrocytic tumors $(9,10)$. In cholangiocarcinoma, immunohistochemistry demonstrated reduced FAT1 expression at the normal membranous location (11) and FAT1 is preferentially downregulated in invasive breast cancer (12). In contrast, FAT1 is upregulated in leukemia and prognosis of precursor B-cell acute lymphoblastic leukemia patients with FAT1 upregulation is poor (13). Functional in vitro studies revealed that FAT1 acts as a regulator of oncogenic pathways in glioma cell lines (14).

Here, we aimed to assess the expression and function of FAT1 in hepatocellular carcinoma (HCC), which is the fifth most common cancer worldwide and the third most common cause of cancer mortality (15). HCC is strongly associated with liver fibrosis, with $90 \%$ of HCC cases arising in cirrhotic livers (16). Recently, we have shown increased FAT1 expression during liver fibrogenesis and identified activated hepatic stellate cells (HSCs) as the cellular source of increased FAT1 expression in diseased livers (17). In the present study, we describe a novel mechanism by which activated HSCs induce FAT1 expression in HCC cells and identify FAT1 as tumor promoter in this highly aggressive hepatic cancer.

\section{Materials and methods}

\section{Cells and cell culture}

The HCC cell lines HepG2 (ATCC HB-8065), PLC (ATCC CRL-8024) and Hep3B (ATCC HB-8064) were cultured as described (18). Primary human hepatocytes (PHH) and HSCs were isolated and cultured as described (18-20). In vitro activation of HSCs was achieved by cell culture on uncoated tissue culture dishes (20). Collection of conditioned medium (CM) from activated HSCs and control medium was done as described (18). For individual experiments, $\mathrm{CM}$ was preincubated with anti-human hepatocyte growth factor (HGF) or isotope control antibodies $(100 \mu \mathrm{g} / \mathrm{ml}$; all from R\&D Systems, Wiesbaden, Germany). Recombinant HGF was purchased from R\&D Systems.

Hypoxia was induced by exposure to $1 \% \mathrm{O}_{2}$ or incubation with 2,2-dipyridyl (DP) (100 $\mu \mathrm{mol} / \mathrm{l}$; Sigma-Aldrich, Deisenhofen, Germany) for the indicated periods of time. For pharmaceutical inhibition of hypoxia-inducible factor (HIF)-1 activity, cells were incubated with $10 \mathrm{nmol} / \mathrm{l}$ of echinomycin (Alexis Biochemicals, Lörrach, Germany). Protein synthesis was inhibited by cycloheximide and for demethylation cells were stimulated with either 5-aza$2^{\prime}$ deoxycytidine (Aza), adenosine-2',3'-dialdehyde (Adox) or $S$-adenosyl-Lmethionine (SAM) (all from Sigma-Aldrich).

Human tissues and HCC tissue microarray

Paired HCC and non-neoplastic liver tissues were obtained from HCC patients undergoing surgical resection. Tissue samples were immediately snap-frozen and stored at $-80^{\circ} \mathrm{C}$ until analysis. A tissue microarray (TMA) of paraffin-embedded HCC samples was constructed as described (18,21-23). Clinicopathological patient characteristics are summarized in Table I.

Tissue samples from liver resections for cell isolation and expression analyses were obtained from patients undergoing partial hepatectomy. Experimental procedures were performed according to the guidelines of the charitable state controlled foundation HTCR (Human Tissue and Cell Research), with the informed patient's written consent approved by the local ethical committee of the University of Regensburg (24). All experiments involving human tissues and cells have been carried out in accordance with The Code of Ethics of the World Medical Association (Declaration of Helsinki).

\section{Transfection of HCC cell lines}

Applying the Lipofectamine plus method (Invitrogen, Carlsbad, CA) cells were transfected with a dominant-negative HIF 1 alpha (HIF1 $\alpha)$ construct (25). FAT1-suppressed cell clones were established by stable transfection of PLC cells with a FAT1 short hairpin RNA (shRNA) plasmid. The FAT1 shRNA plasmid was prepared as shown previously (26). Plasmids were cotransfected with pcDNA3 (Invitrogen), containing the selectable marker for neomycin resistance. Controls received pcDNA3 alone. One day after transfection, cells 
Table I. FAT1 immunoreactivity (IR) in HCC tissue of 112 patients in relation to clinicopathological characteristics and proliferation rate

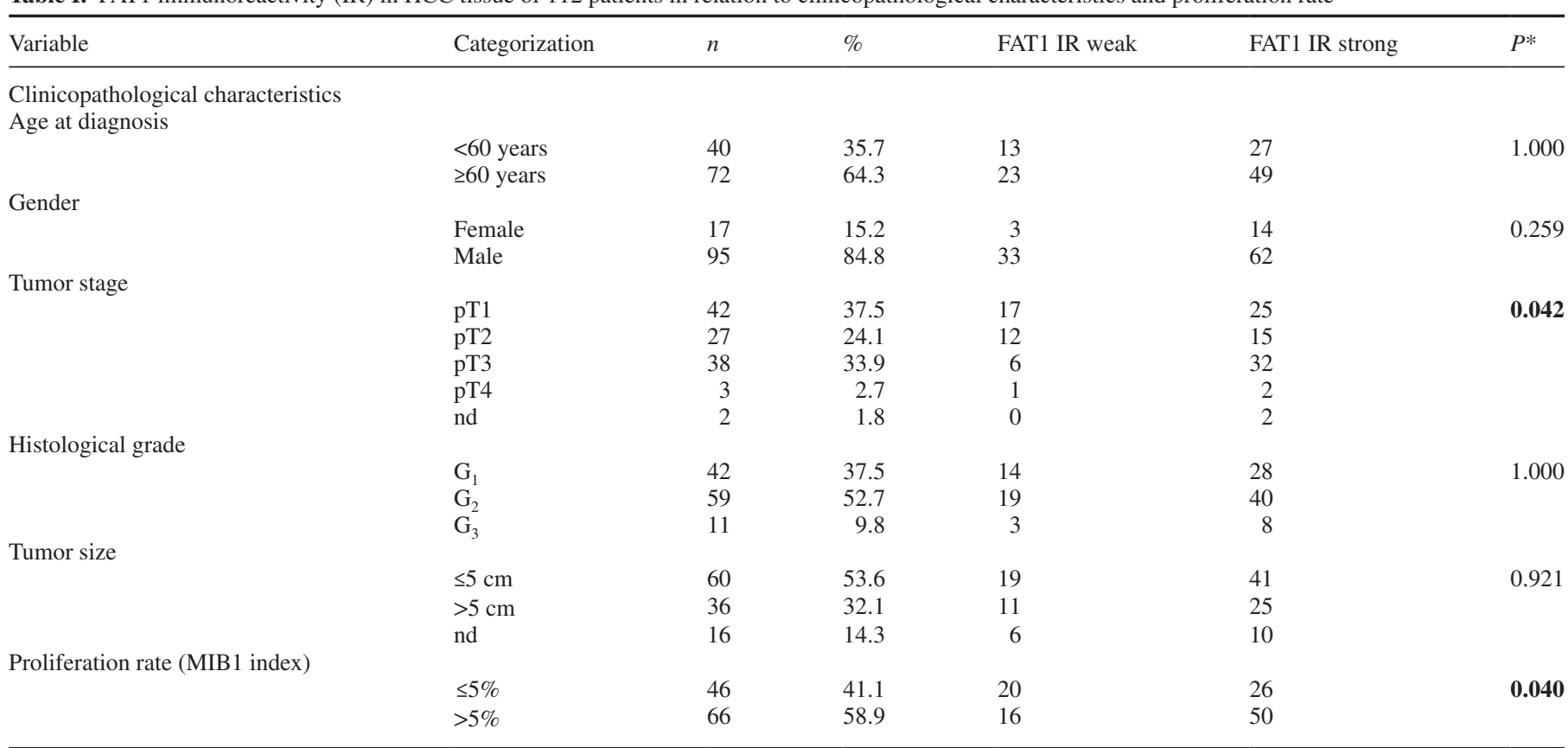

IR, immunoreactivity; nd, no data available.

*Fisher's exact test (two sided); boldface representing $P$ values $<0.05$.

were placed in selection medium containing $200 \mu \mathrm{g} / \mathrm{ml}$ G418 (Sigma). After 25 days of selection, individual G418-resistant colonies were subcloned.

\section{AP-1 reporter gene assay}

Cells were transfected with a AP1 luc plasmid (Stratagene, La Jolla, CA) using Lipofectamine Plus reagent (Invitrogen, Karlsruhe, Germany) as described (27). For normalization of transfection efficiency, cells were contransfected with pRL-TK plasmid resulting in a renilla luciferase acitivity (Promega, Mannheim, Germany), and after $24 \mathrm{~h}$ cells were lysed and the luciferase activities were measured as described (27).

\section{Expression analysis}

Isolation of total cellular RNA from cultured cells and tissues and reverse transcription were performed as described previously. Quantitative real-time PCR was performed on a LightCycler (Roche, Mannheim, Germany) using the following sets of primers: FAT1 (for: 5'-GTG TTT GTT CTC TGC CGT AAG-3'; rev: 5'TAG GCT TCT GGA TGG AGT CG-3'), E-CAD (for: 5'-ATC CTC CGA TCT TCA ATC CCA CCA C-3'; rev: 5'-GTA CCA CAT TCG TCA CTG CTA CGT G-3'), MAT2A (for: 5'-CCA CGA GGC GTT CAT CGA GG-3'; rev: 5'-AAG TCT TGT AGT CAA AAC CT-3'). Vascular endothelial growth factor (VEGF), SNAIL, Myc-associated zinc finger protein (MAZ) and PDCD4 messenger RNA (mRNA) expression analysis were performed using QuantiTect Primer Assay according to the manufacturer's instructions (Qiagen, Hilden, Germany).

Protein analysis

Protein extraction and western blotting were performed as described (18) using the following antibodies: anti-FAT1 (1:1000; Atlas Antibodies AB, Stockholm, Sweden), anti-HIF1 $\alpha$ (1:200; Santa Cruz Biotechnology, Santa Cruz, CA) and anti- $\beta$-actin (1:20 000; Sigma).

For immunohistochemistry, hematoxylin and eosin staining, and terminal deoxynucleotidyl transferase-mediated dUTP nick end labeling staining, standard $5 \mu \mathrm{m}$ sections of formalin-fixed and paraffin-embedded tissue blocks were used.

Immunohistochemical staining was performed using the following antibodies: anti-FAT1 (1:50; Atlas Antibodies AB), Ki67 (MIB1, 1:50; Abcam, Cambridge, UK), Caspase 3 (1:150; Epitomics, Burlingame, CA) and anti-cJun (1:100, \#9165; Cell Signaling Technology), as described (28). Terminal deoxynucleotidyl transferase-mediated dUTP nick end labeling staining was performed as described (21).

For analysis of the TMA, positivity for FAT1 was defined as detectable membranous staining, whereas cases designated as FAT1 negative were devoid of FAT1 staining.

S-Adenosyl-L-methionine extraction and analysis

For analysis of $S$-adenosyl-L-methionine (SAM) in cell culture medium, cells were cultured in serum-free medium for $24 \mathrm{~h}$. Subsequently, medium was collected, centrifuged, and the supernatant was snap-frozen and stored at $-80^{\circ} \mathrm{C}$. SAM analysis was performed with liquid chromatography-electrospray ionization-tandem mass spectrometry as described (29).

Analysis of proliferation, migration and resistance against sorafenib treatment

Cell proliferation was measured using the XTT (2,3-bis-(2-methoxy-4-nitro5-sulfophenyl)-2H-tetrazolium-5-carboxanilide) assay (Roche) as described (22). Cell migration was quantified using the xCELLigence system according to the instructions of the supplier (Roche Applied Science).

To analyze the resistance against sorafenib treatment, cells were incubated with different doses of sorafenib $(3,10,30 \mu \mathrm{M})$ or vehicle (dimethyl sulfoxide) for $48 \mathrm{~h}$ followed by analysis of XTT activity.

Analysis of apoptosis

For detection of apoptosis, cells were stained simultaneously with FITCconjugated Annexin V and propidium iodide (both from Pharmingen, Germany) and analyzed by flow cytometry (20). Further, the Caspase-Glo 3/7 Assay (Promega, Madison, WI) was used to analyze caspase-3/7 activity (30).

Tumor cell inoculation and measurement of tumor growth in NMRI $(n u / n u)$ mice

A model of inoculation of tumor cells into NMRI (nu/nu) mice to monitor tumor growth in vivo was performed as described $(21,22)$ following the institutional (University of Regensburg) and National Guidelines for the Care and Use of Animals. For ethical reasons, mice were killed upon observation of the first signs of tumor ulceration. Tumors were excised, snap-frozen and stored in liquid nitrogen for subsequent analysis.

Statistical analysis

Results are expressed as mean \pm standard error (range) or percent. Comparison between groups was made using the Student's unpaired $t$-test. A $P$ value $<$ 0.05 was considered statistically significant. Contingency table analysis and the two-sided Fisher's exact test were used to study the statistical association between clinicopathological and immunohistochemical variables. All calculations were performed by using the GraphPad Prism Software (GraphPad Software, San Diego, CA) or SPSS (SPSS, Chicago, IL).

\section{Results}

FAT1 expression in HCC

Initially, we analyzed FAT1 mRNA expression in three different HCC cell lines (HepG2, PLC and Hep3B) and PHH by quantitative realtime PCR and western blot analysis. In all three HCC cell lines FAT1 
A

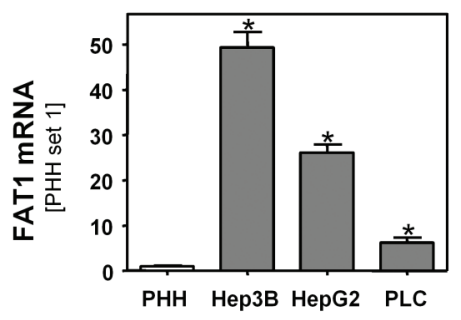

C

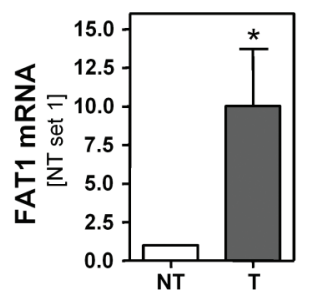

B

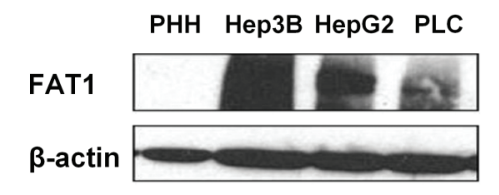

D
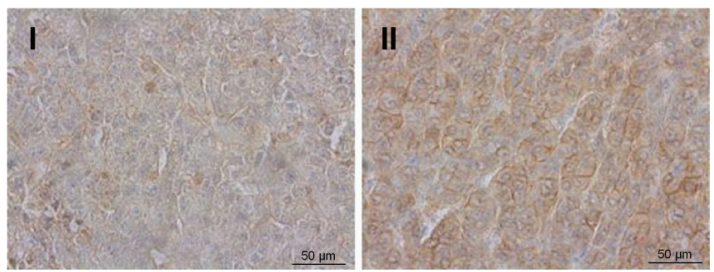

Fig. 1. FAT1 expression in HCC. (A) mRNA and (B) protein expression of FAT1 in HCC cell lines (HepG2, PLC and Hep3B) and PHH. (C) FAT1 mRNA expression in tumor tissue of 14 HCC patients (T) and in corresponding non-neoplastic liver (NT). (D) Examples of weak (I) and strong (II) immunohistochemical FAT1 staining of human HCC tissues. ( ${ }^{*} P<0.05$ compared with PHH or NT).

mRNA and protein expression were significantly increased compared with PHH (Figure 1A and B). Comparison of human HCC tissue and corresponding non-tumorous liver tissue of 14 patients confirmed a strong upregulation of FAT1 mRNA in cancerous tissue (Figure 1C).

To evaluate the functional effects of FAT1 upregulation in HCC in vivo, we analyzed FAT1 protein expression in a series of 112 human HCC tissues, applying immunohistochemistry and TMA technology. Membranous FAT1 staining intensity varied significantly among individual patients. For descriptive data analysis, HCC were scored as exhibiting either strong or weak immunosignal (representative examples are depicted in Figure 1D), and immunohistochemical results were correlated with clinicopathological characteristics (Table I). Strong membranous FAT1 staining was significantly associated with higher tumor stage $(P=0.042)$ and proliferation rate $(K i-67$ labelling index; $P=0.040)$, respectively. No correlation was found between FAT1 expression and tumor grading and tumor size or patients' age and sex.

\section{Inhibition of FAT1 expression in HCC cells}

To gain insight into the functional role of increased FAT1 in HCC, we inhibited FAT 1 expression in the HCC cell line PLC by stable transfection with a shRNA expression vector containing the sequence of FAT1 small interfering RNA (siRNA) (FAT1 shRNA\#1, FAT1 shRNA\#2). PLC cells transfected with the empty vector served as control. Quantitative reverse transcription-polymerase chain reaction and western blot analysis revealed a strong downregulation of FAT1 expression in FAT1 shRNA compared with control cell clones (Figure 2A). FAT1-suppressed HCC cells showed normal histological morphology (Figure 2B), and also the expression of E-Cadherin (E-Cad) and Snail, two markers of epithelialmesenchymal transition, did not differ significantly compared with control cells (Supplementary Figure 1, available at Carcinogenesis Online).

To characterize the role of FAT1 in HCC cells, we performed functional in vitro assays with FAT1-suppressed cells in comparison with control cells. HCC cells with suppressed FAT1 expression grew significantly slower (Figure 2C). Migration assays demonstrated that suppression of FAT1 inhibited the migratory potential of HCC cells (Figure 2D). Moreover, serum starvation induced significantly higher caspase-3/7 activation (Figure 2E) and a higher apoptosis rate in HCC cells with suppressed FAT1 expression compared with control cells (Figure 2F). We also analyzed the resistance of FAT1 suppressed and control cells against sorafenib treatment in vitro but did not observe significant differences (Figure 2G).
In search for the underlying mechanism of FAT1 on proliferation, migration and apoptosis resistance in HCC cells, we analyzed expression of tumor suppressor gene programmed cell death 4 (PDCD4) because a recent study by Dikshit et al. (14) identified FAT1 as inhibitor of this tumor suppressor gene in glioblastoma multiforme. Also in HCC PDCD4 was identified as tumor suppressor gene inducing apoptosis and inhibiting their metastatic potential $(31,32)$. However, PDCD4 expression levels did not differ significantly between FAT1suppressed and control cells (Supplementary Figure 2A, available at Carcinogenesis Online). Moreover, Dikshit et al. discovered that FAT1 inhibited c-Jun phosphorylation and herewith led to the attenuation of activator protein-1 (AP-1) in glioblastoma. AP-1 is a critical regulator of tumorigenicity of HCC cells $(33,34)$ but reporter gene analysis showed similar activity in HCC cells with and without FAT1 suppression (Supplementary Figure 2B, available at Carcinogenesis Online).

\section{Tumorigenicity of FAT1-suppressed HCC cells in vivo}

To test the effect of FAT1 on tumor growth in vivo, HCC cells stably suppressing FAT1 and control cells were injected subcutaneously into nude mice. Tumors derived from FAT1-suppressing HCC cell clones showed delayed tumor onset compared with tumors derived from mock-transfected control cells (Figure 3A). Expression analysis revealed preservation of FAT1 mRNA suppression in tumors (Figure 3B). Terminal deoxynucleotidyl transferase dUTP nick-end labeling staining showed only few apoptotic cells in control-transfected HCC-derived tumors, whereas the FAT1-suppressing tumors displayed large apoptotic areas (Figure 3C).

\section{Regulation of FAT1 expression in HCC cells}

Activated HSCs form and transduce the HCC stroma (35). Previously, we have shown that HSCs secrete factors by which they promote growth and migration of HCC cells in vitro (36). This prompted us to examine the effect of CM from activated HSCs on FAT1 mRNA expression in HCC cells. Quantitative reverse transcription-polymerase chain reaction showed increased FAT1 mRNA expression in $\mathrm{HCC}$ cells after incubation with CM from HSCs (Figure 4A). In search for the factors in CM of HSCs responsible for FAT1 upregulation in HCC cells we focused on HGF, which is known to promote hepatocancerogenesis. 
A

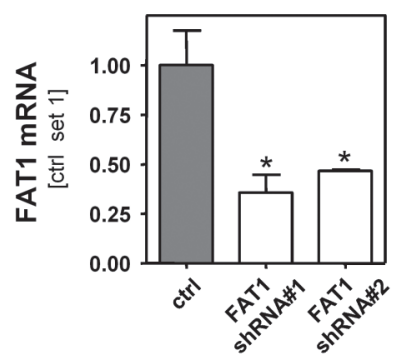

B
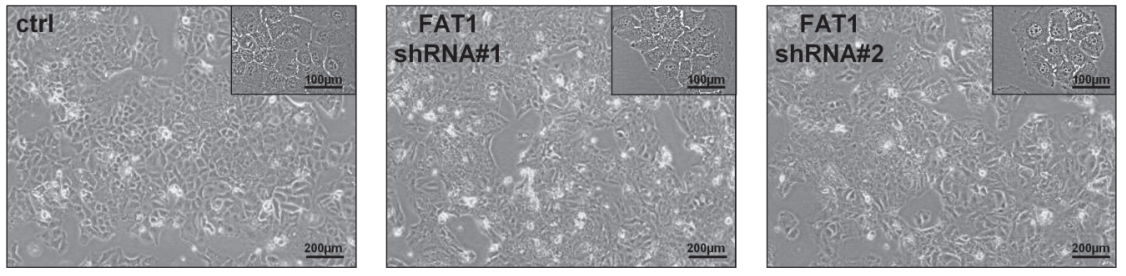

C

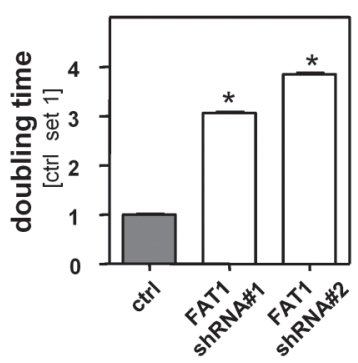

E

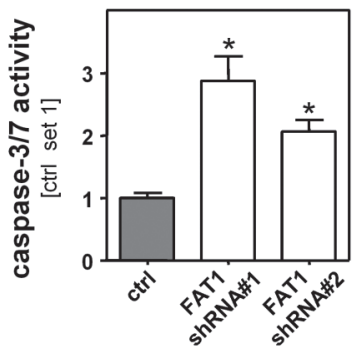

FAT1

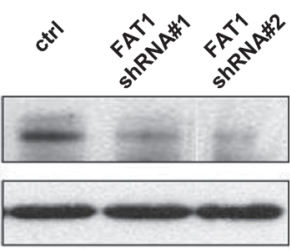

$\beta$-actin
D

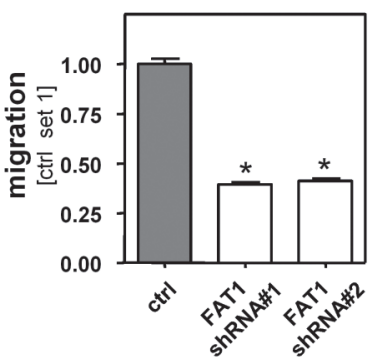

F

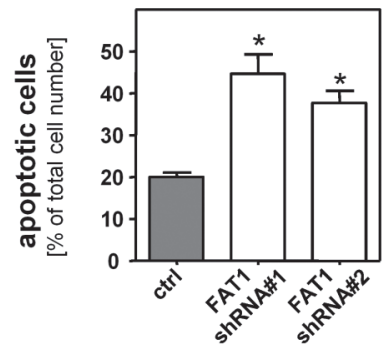

G

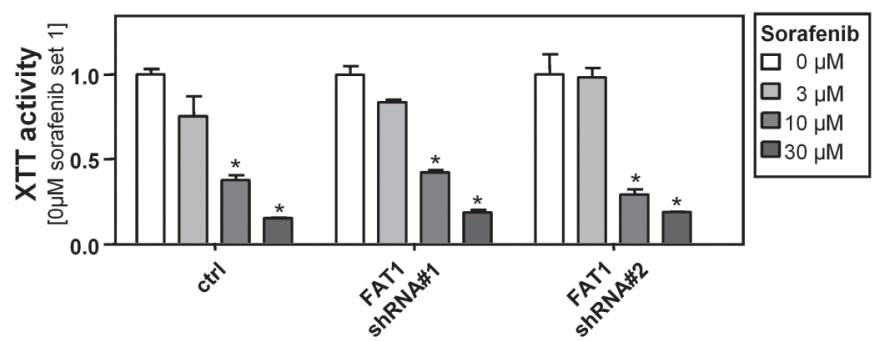

Fig. 2. Inhibition of FAT1 expression in HCC cells. (A) FAT1 mRNA and protein expression in HCC cell clones stably transfected with FAT1 shRNA (FAT1 shRNA\#1; FAT1 shRNA\#2) or control shRNA (ctrl) $(* P<0.05$ compared with ctrl). (B) Morphology of FAT1-suppressed and control cells. (C) Doubling time and (D) migration of FAT1-suppressed and control cells $(* P<0.05$ compared with ctrl). (E) Analysis of caspase 3/7 activity in FAT1-suppressed and control cells after serum deprivation $(* P<0.05$ compared with ctrl). (F) Assessment of apoptosis by flow cytometry applying annexin $\mathrm{V}$ and propidium iodide (PI) staining. Depicted is the mean percentage of total apoptotic cells from three independent experiments ( $* P<0.05$ compared with ctrl). (G) Analysis of XTT activity in FAT1-suppressed and control cells after incubation with sorafenib $(3,10,30 \mu \mathrm{M})$ in relation to control cells without sorafenib treatment $(* P<0.05$ compared with cells without sorafenib treatment).

Stimulation of HCC cells with HGF (50 ng/ml) induced a significant upregulation of FAT1 mRNA and protein expression (Figure 4B). Preincubation of CM from activated HSCs with anti-HGF antibodies completely abrogated the stimulating effect on FAT1 (Figure 4C). Together, these data indicate that activated HSCs induce FAT1 in HCC cells via HGF secretion. 
A

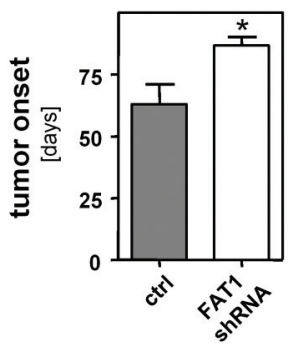

C
B
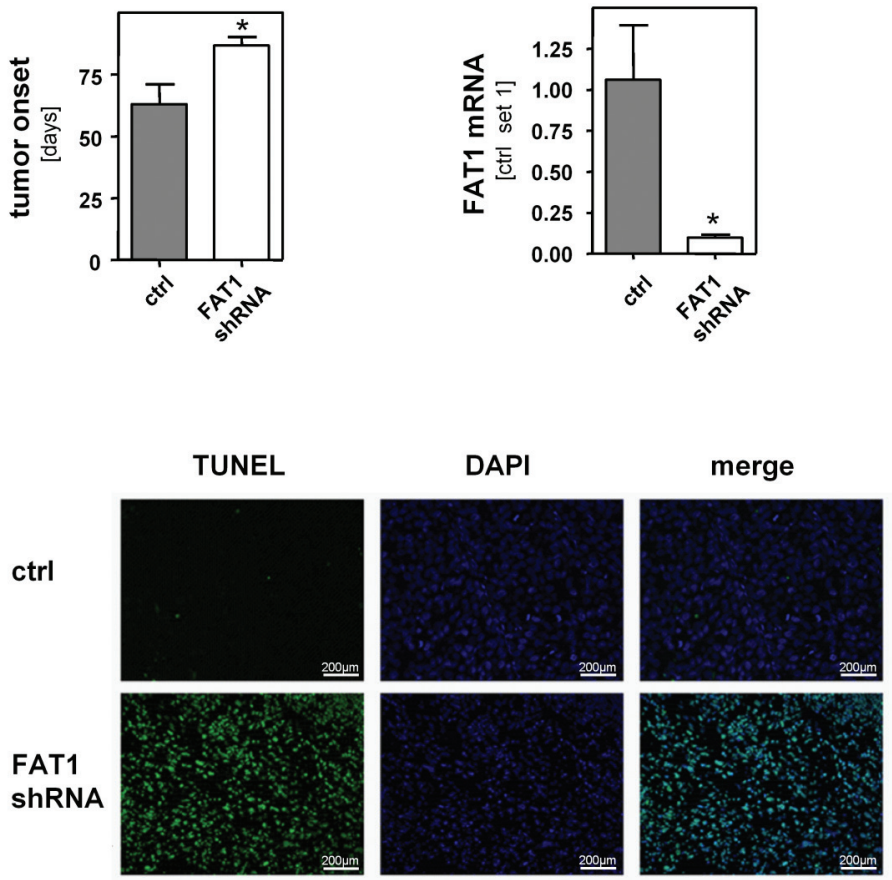

Fig. 3. Tumorigenicity of FAT1-suppressed HCC cells in vivo. (A) Onset of tumors derived from FAT1-suppressed or control cells implanted in nude mice. (B) FAT1 mRNA expression in tumors derived from FAT1-suppressed or control cells. (C) Analysis for apoptotic cells in tumor tissue using terminal deoxynucleotidyl transferase-mediated dUTP nick end labeling (TUNEL) staining (green) and 4',6-diamidino-2-phenylindole (DAPI) staining for nuclei (blue) $(* P<0.05$ compared with ctrl).

To further elucidate the HGF-induced transcriptional regulation of FAT1 we focused on HIF1 $\alpha$, because it has been shown that HGF enhances the activity of this transcription factor in HCC cells (37). Further, HIF1 $\alpha$ plays a critical role in HCC development and progression (38). HGF-induced FAT1 expression was completely inhibited by echinomycin, a pharmaceutical inhibitor of HIF1 $\alpha$ activity (Figure 4D). HIF1 $\alpha$ is also an important mediator of hypoxic adaption of tumor cells (39), and accordingly, induction of hypoxia by incubation with the chemical inducer DP caused a significant upregulation of FAT1 (Figure 4E and F). Western blot analysis demonstrated stabilization of HIF1 $\alpha$ in DP-treated cells (Figure 4F). FAT1 upregulation under DP-induced hypoxia was strongly repressed by echinomycin (Figure 4G). In line with this finding, transient transfection with a dominant-negative variant of HIF1 $\alpha(40)$ led to diminished DP-induced FAT1 expression (Figure 4H). Together, these data indicate HIF1 $\alpha$ as critical regulator of HGF- and hypoxia-induced FAT1 expression in HCC cells.

\section{Mechanisms of FAT1 regulation in HCC}

However, in silico analysis of the FAT1 promoter did not show an HIF1 binding site [Tess (http://www.cbil.upenn.edu/cgi-bin/tess/ tess?RQ=WELCOME) and Genomatix software; data not shown]. Furthermore, costimulation with cycloheximide, an inhibitor of protein synthesis, abolished HGF- and hypoxia-induced upregulation of FAT1 mRNA expression in HCC cells (Figure 5A and Supplementary Figure 2, available at Carcinogenesis Online). These data indicated that hypoxia and HIF1 $\alpha$, respectively, did not directly induce FAT1 expression at the transcriptional level. Still, hypoxia can cause epigenetic modifications as demethylation (41), and it has been shown that the methylation status of the FATl $\mathrm{CpG}$ island in squamous cell carcinomas correlated negatively with its expression (10). Here, we found that incubation with 5-aza-2'deoxycytidine (Aza) and adenosine-2', $3^{\prime}$-dialdehyde (Adox) both inhibitors of DNA methylation, caused a dose-dependent upregulation of FAT1 expression in HCC cells (Figure 5B). Most recently, it has been shown that hypoxia-induced DNA demethylation in HCC is caused through activation of HIF1 $\alpha$ and transcriptional upregulation of methionine adenosyltransferase II, alpha (MAT2A) (42). MAT is the critical enzyme for the reaction of methionine and adenosine triphosphate to SAM. There are two genes (MAT1A, MAT2A) for two homologous catalytic MAT subunits (MATI, MATII). Although MAT1A is mainly expressed in healthy liver, a switch from MAT1A to MAT2A takes place during the progression of HCC (43). This leads to reduced SAM levels due to the lower enzymatic activity of MATII compared with MATI (44). Also in the present study we observed reduced SAM levels in the supernatant of HCC cells after chemical induction of hypoxia (Figure 5C). Replenishment of SAM in culture medium of HCC cells dose-dependently inhibited hypoxia-induced FAT1 expression (Figure 5D). These findings indicate that hypoxia-induced FAT1 expression in HCC cells is mediated via MAT2A-mediated SAM depletion and subsequent demethylation of the FAT1 promotor.

To verify these findings in vivo we assessed FAT1 and VEGF expression in 25 human HCC specimens by quantitative reverse transcription-polymerase chain reaction. VEGF is highly regulated by HIF1 $\alpha$ in HCC (45), and notably, we found a significant correlation between VEGF and FAT1 expression in HCC tissues (Figure 5E). Furthermore, FAT1 and MAT2A expression showed a significant correlation in HCC tissues (Figure 5F). Together, these data suggest that also in vivo hypoxia is a critical regulator of FAT1 expression in HCC. This effect seems to be at least in part mediated via enhanced MAT2A expression and subsequent depletion of SAM levels leading to demethylation of the FAT1 promoter.

In search for the molecular mechanisms responsible for the significantly enhanced basal FAT1 expression levels in HCC cells compared with hepatocytes, we focused on MAZ because in silico analysis (Genomatix software) revealed a MAZ binding site located $242 \mathrm{bp}$ upstream of the transcriptional start site of the human FAT1 gene. Furthermore, we have shown previously that $M A Z$ expression in HCC cells is markedly enhanced compared with PHH (18). Transient 
A

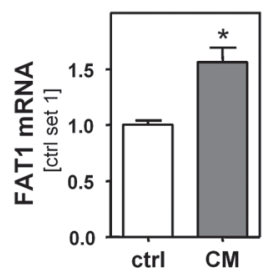

C

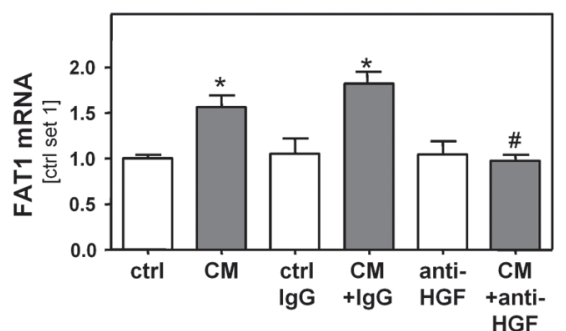

E

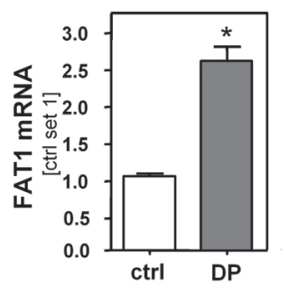

G

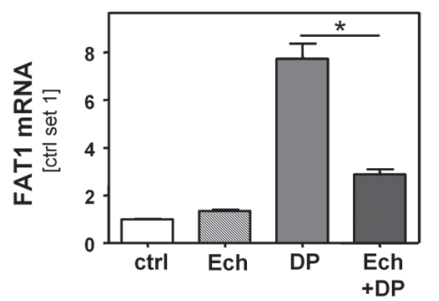

B

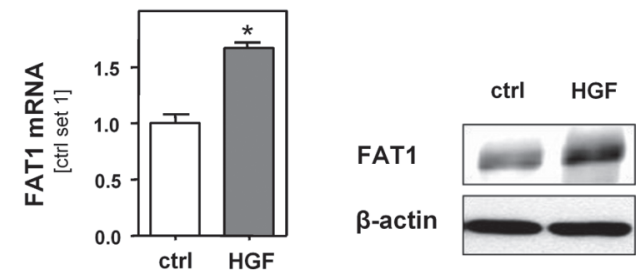

D

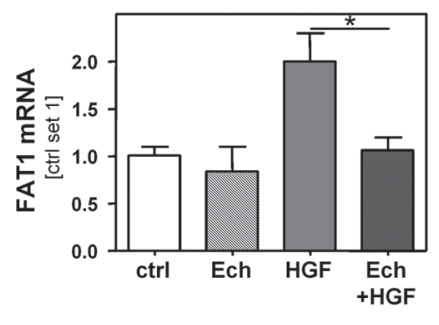

F

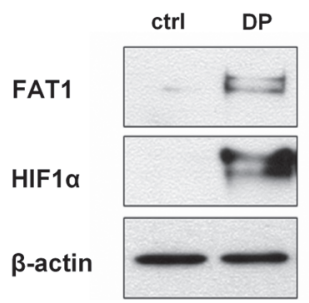

H

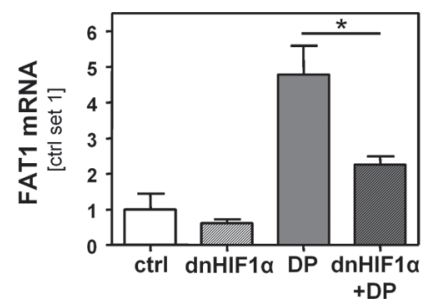

Fig. 4. Regulation of FAT1 in HCC cells. (A) Analysis of FAT1 mRNA expression in HCC cells after incubation with control medium (ctrl) and CM of HSCs. (B) Analysis of FAT1 mRNA and protein expression after incubation with HGF (50 ng/ml). (C) Effect of anti-HGF antibodies on FAT1 mRNA expression in the presence of CM. CM was preincubated with anti-HGF antibodies or isotype-matched control antibodies (ctrl IgG) before addition to HCC cells $(* P<0.05$ compared with ctrl; ${ }^{\#} P<0.05$ compared with CM). (D) FAT1 mRNA expression in HGF-stimulated cells with or without inhibiting HIF1 $\alpha$ with echinomycin (Ech). (E) Analysis of FAT1 mRNA expression in HCC cells after pharmaceutical induction of hypoxia by DP. (F) FAT1 and HIF1 $\alpha$ protein expression after chemical induction of hypoxia by DP. (G) FAT1 mRNA expression with or without pharmaceutical induction of hypoxia and HIF1 $\alpha$ inhibition with Ech by quantitative PCR. (H) Analysis of FAT1 mRNA after transfection of HCC cells with a dominant-negative variant of HIF1 $\alpha(\mathrm{dnHIF} 1 \alpha)$ with or without stimulation with DP $(* P<0.05$ compared with DP $)$.

transfection with two different MAZ siRNAs significantly inhibited MAZ mRNA expression in Hep3B cells compared with HCC cells transfected with control siRNA (ctrl siRNA) and non-transfected control cells (ctrl) (Figure 5G). Notably, MAZ suppression caused a significant but not complete downregulation of FAT1 expression in HCC cells (Figure 5H).

In summary, these data indicate that constitutively high FAT1 mRNA expression in HCC cells under normoxic conditions is at least in part dependent on the transcription factor MAZ. FAT1 mRNA expression is further increased via HSC-secreted HGF or hypoxiainduced HIF-1 activation in HCC.

\section{Discussion}

The giant cadherin FAT1 is one of four vertebrate orthologues of the Drosophila tumor suppressor fat. The specific function of FAT1 in cancer development and progression is still under investigation. There are contrasting studies about the role of FAT1 in human cancers, pointing toward a dual role of FAT1 as both an oncogene as well as a tumor suppressor $(9-13,46)$. Here, we found increased FAT1 expression in HCC cells and tissues compared with primary human hepatocytes and non-tumorous liver tissue. Furthermore, FAT1 expression correlated with higher tumor stage and mitotic activity in human HCCs. These 
A

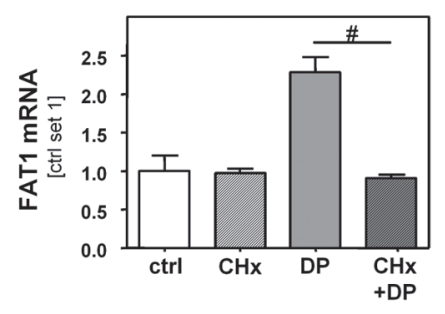

C

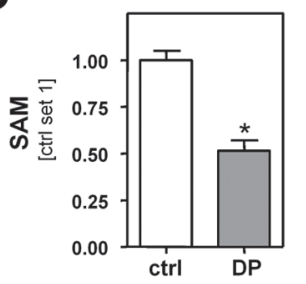

E

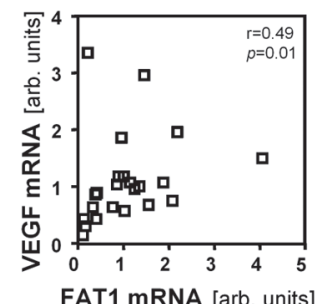

G

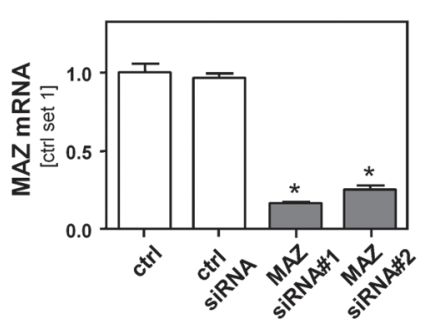

B

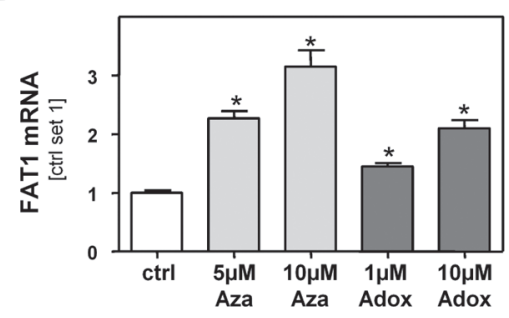

D

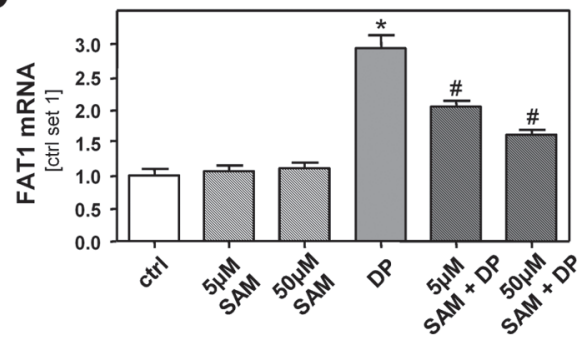

F

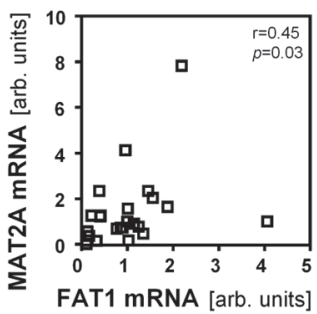

H

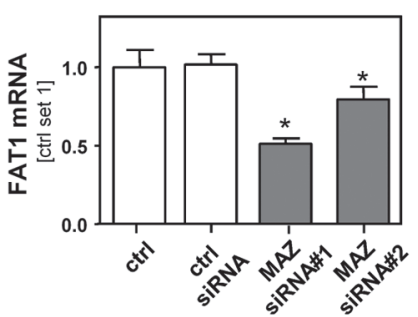

Fig. 5. Mechanisms of FAT1 regulation in HCC. (A) FAT1 mRNA expression in DP-stimulated cells with or without blocking protein synthesis by cycloheximide (CHx) $\left({ }^{\#} P<0.05\right)$. (B) Analysis of FAT1 mRNA expression in HCC cells after treatment with different doses of 5-aza-2'deoxycytidine (Aza) and adenosine- $2^{\prime}, 3^{\prime}$-dialdehyde (Adox) for $48 \mathrm{~h}(* P<0.05)$. (C) SAM level after induction of hypoxia with DP analyzed by liquid chromatography-electrospray ionization-tandem mass spectrometry $(* P<0.05)$. (D) FAT1 mRNA expression in HCC cells stimulated with different doses of SAM and subsequent induction of hypoxia with DP for $48 \mathrm{~h}\left({ }^{*} P<0.05\right.$ compared with ctrl; ${ }^{\#} P<0.05$ compared with DP). (E) Correlation of FAT1 with VEGF and (F) MAT2A mRNA expression in HCC tissue $(n=24)$. (G) Analysis of MAZ and (H) FAT1 mRNA expression in HCC cells transiently transfected with two different MAZ siRNAs (siRNA\#1 and siRNA\#2) or control siRNA (ctrl siRNA) and non-transfected control cells (ctrl) $\left({ }^{*} P<0.05\right.$ compared with ctrl siRNA).

findings indicated that FAT1 acts oncogenic in HCC, and in functional studies we identified a protumorigenic effect of FAT1 in HCC cells. We observed reduced migratory potential in HCC cells upon FAT1 suppression in concordance with studies on squamous cell carcinoma and glioma cells (9-14). In breast cancer, FAT1 seems to inhibit progression to an invasive phenotype (47). In cholangiocarcinoma, FAT1 expression showed an inverse correlation with the proliferation index and loss of membranous FAT1 localization correlated with more aggressive tumor growth (11). In non-malignant vascular smooth muscle cells FAT1 knockdown lead to decreased migratory activity but surprisingly and in contrast to our findings in HCC cells enhanced proliferation (48). In addition to its varying effects on proliferation and migration in different cell types, FAT1 is engaged in several other biological functions such as polarity and adhesion or as a regulator of inflammatory pathways in cancer cells (49). However, available literature has not yet functionally linked FAT1 to apoptosis. Here, we demonstrate that FAT1 downregulation impairs the resistance of HCC cells against induced apoptosis in vitro. Also in vivo tumors derived from HCC cells with repressed FAT1 expression revealed significantly more apoptosis than tumors derived from control HCC cells. In summary, our data indicate FAT1 as tumor promotor in HCC. Differently than observed in glioblastoma multiforme (14), we did not observe that FAT1 affected PDCD4 expression levels or AP-1 activity in HCC cells. Currently, we can only speculate on the protumorigenic molecular mechanisms set in motion by FAT1 expression in HCC. A most recent review by Sadeqzadeh et al. (50) highlights that we are just beginning 
to understand the molecular interactions of FAT1, which vary in different tissues and in health and disease. Future studies have to identify the mechanism by which FAT1 exhibits protumorigenic effects in HCC.

In this study we focused on the molecular mechanism responsible for the transcriptional upregulation of FAT1 in HCC. Here, we investigated effects mediated by activated HSCs because these cells form and infiltrate the HCC stroma and affect HCC development and progression $(16,36,46,51,52)$. Furthermore, we recently identified activated HSCs as the cellular source of increased FAT1 expression in liver fibrosis and demonstrated that FAT1 is a profibrogenic factor in these cells (17). Due to these profibrogenic effects in HSCs and due to the here newly described tumorigenic effects, FAT1 might constitute an excellent therapeutic target in chronic liver disease. Moreover, we found that activated HSCs induce FAT1 expression in HCC cells underscoring the critical role of activated HSCs in hepatocancerogenesis (16).

Interestingly, activated HSCs induce FAT1 in HCC cells via secreted HGF. This multifunctional growth factor and its receptor and its high-affinity tyrosine kinase receptor c-MET are critical regulators of HCC development and progression (53). We found that the HGF effect on FAT1 activation is mediated via increased activation of HIF1 $\alpha$, which plays a critical role in hepatocancerogenesis (38) In addition to HGF, hypoxia typically stabilizes HIF1 $\alpha$ and herewith leads to an activation of this pathway. Hypoxic conditions frequently occur in tumorous tissue and are another critical promoter of HCC progression (39). Interestingly, we found that HIF1 $\alpha$ effects on FAT1 expression in HCC cells were not mediated directly at the transcriptional level but via reduction of the levels of the methyl donor SAM. Liu et al. (42) identified HIF1 $\alpha$-mediated upregulation of MAT2A as a novel mechanism, by which hypoxia induces DNA demethylation. We confirmed a significant correlation between FAT1 and MAT2A in HCC tissues. Together, these findings lead to the following model of FAT1 regulation in HCC cells (Supplementary Figure 4, available at Carcinogenesis Online): HGF-induced and hypoxia-mediated HIF1 $\alpha$ activation enhances MAT2A expression and herewith MATII activity. As a result MATI activity is reduced, leading to lower levels of the methyl donor SAM. Lower SAM levels cause reduced methylation of the FAT1 promoter and thereby higher FAT1 expression.

A previous study described the regulatory role of SAM in HGFmediated hepatocytes proliferation through a mechanism that implicated the activation of the non-canonical LKB1/AMPK/eNOS cascade and the function of human antigen $\mathrm{R}(\mathrm{HuR})$, which stabilizes MAT2A mRNA (54). In HCC cells we identified a different regulatory pathway by which $\mathrm{HGF}$ - and also hypoxia - affected SAM levels, and herewith, FAT1 promoter methylation. Thus, this study identifies an example how signaling pathways propagated from the cell surface through transmembrane receptors to intracellular regulatory mechanisms are critical for normal as well as aberrant cellular functions. This knowledge may provide the basis for the rational design of novel therapeutics to inhibit HCC development and progression.

\section{Supplementary material}

Supplementary Figures 1-4 can be found at http://carcin. oxfordjournals.org/

\section{Funding}

German Research Foundation (DFG) to P.O., A.B. and C.H. (KFO 262); German Ministry of Education and Research (BMBF) to A.B. and C.H

\section{Acknowledgements}

We are indebted to K.Elser, B.Ott-Rötzer and R.Jung for excellent technical assistance. Furthermore, we thank C.Warnecke (Erlangen, Germany) for providing the dnHIF1 $\alpha$ plasmid. We acknowledge the Human Tissue and Cell Research (HTCR) Foundation for supporting our research by making human liver tissue available.
Conflict of Interest Statement: None declared.

\section{References}

1. Halbleib,J.M. et al. (2006) Cadherins in development: cell adhesion, sorting, and tissue morphogenesis. Genes Dev., 20, 3199-3214.

2. Berx, G. et al. (2009) Involvement of members of the cadherin superfamily in cancer. Cold Spring Harb. Perspect. Biol., 1, a003129.

3. Tanoue,T. et al. (2005) New insights into fat cadherins. J. Cell Sci., $118(\mathrm{Pt}$ 11), 2347-2353.

4. Matakatsu,H. et al. (2004) Interactions between fat and Dachsous and the regulation of planar cell polarity in the Drosophila wing. Development, 131, 3785-3794.

5. Dunne,J. et al. (1995) Molecular cloning and tissue expression of FAT, the human homologue of the Drosophila fat gene that is located on chromosome 4q34-q35 and encodes a putative adhesion molecule. Genomics, 30, 207-223.

6. Ciani,L. et al. (2003) Mice lacking the giant protocadherin mFAT1 exhibit renal slit junction abnormalities and a partially penetrant cyclopia and anophthalmia phenotype. Mol. Cell. Biol., 23, 3575-3582.

7. Braun,G.S. et al. (2007) Differentially spliced isoforms of FAT1 are asymmetrically distributed within migrating cells. J. Biol. Chem., 282, 22823-22833.

8. Katoh,M. (2012) Function and cancer genomics of FAT family genes (review). Int. J. Oncol., 41, 1913-1918.

9. Chosdol,K. et al. (2009) Frequent loss of heterozygosity and altered expression of the candidate tumor suppressor gene 'FAT' in human astrocytic tumors. BMC Cancer, $9,5$.

10. Nakaya,K. et al. (2007) Identification of homozygous deletions of tumor suppressor gene FAT in oral cancer using CGH-array. Oncogene, 26, 5300-5308.

11. Settakorn,J. et al. (2005) FAT, E-cadherin, beta catenin, HER 2/neu, Ki67 immuno-expression, and histological grade in intrahepatic cholangiocarcinoma. J. Clin. Pathol., 58, 1249-1254.

12. Kwaepila,N. et al. (2006) Immunohistological localisation of human FAT1 (hFAT) protein in 326 breast cancers. Does this adhesion molecule have a role in pathogenesis? Pathology, 38, 125-131.

13. de Bock,C.E. et al. (2012) The Fat1 cadherin is overexpressed and an independent prognostic factor for survival in paired diagnosis-relapse samples of precursor B-cell acute lymphoblastic leukemia. Leukemia, 26, 918-926.

14. Dikshit,B. et al. (2013) FAT1 acts as an upstream regulator of oncogenic and inflammatory pathways, via PDCD4, in glioma cells. Oncogene, 32, 3798-3808.

15. Shariff,M.I. et al. (2009) Hepatocellular carcinoma: current trends in worldwide epidemiology, risk factors, diagnosis and therapeutics. Expert Rev. Gastroenterol. Hepatol., 3, 353-367.

16.Zhang,D.Y. et al. (2012) Fibrosis-dependent mechanisms of hepatocarcinogenesis. Hepatology, 56, 769-775.

17. Valletta,D. et al. (2012) Expression and function of the atypical cadherin FAT1 in chronic liver disease. Biochem. Biophys. Res. Commun., 426 , 404-408.

18. Amann,T. et al. (2009) GLUT1 expression is increased in hepatocellular carcinoma and promotes tumorigenesis. Am. J. Pathol., 174, 1544-1552.

19. Weiss,T.S. et al. (2003) Cellular damage to human hepatocytes through repeated application of 5-aminolevulinic acid. J. Hepatol., 38, 476-482.

20. Mühlbauer,M. et al. (2006) PD-L1 is induced in hepatocytes by viral infection and by interferon-alpha and -gamma and mediates T cell apoptosis. $J$. Hepatol., 45, 520-528.

21. Amann,T. et al. (2010) Reduced expression of fibroblast growth factor receptor 2IIIb in hepatocellular carcinoma induces a more aggressive growth. Am. J. Pathol., 176, 1433-1442.

22. Hellerbrand,C. et al. (2008) The novel gene MIA2 acts as a tumour suppressor in hepatocellular carcinoma. Gut, 57, 243-251.

23. Kirovski,G. et al. (2011) Down-regulation of methylthioadenosine phosphorylase (MTAP) induces progression of hepatocellular carcinoma via accumulation of 5'-deoxy-5'-methylthioadenosine (MTA). Am. J. Pathol., 178, $1145-1152$.

24. Thasler,W.E. et al. (2003) Charitable state-controlled foundation human tissue and cell research: ethic and legal aspects in the supply of surgically removed human tissue for research in the academic and commercial sector in Germany. Cell Tissue Bank., 4, 49-56.

25. Maegdefrau,U. et al. (2009) Bone morphogenetic protein 4 is induced in hepatocellular carcinoma by hypoxia and promotes tumour progression. $J$. Pathol., 218, 520-529.

26. Shukla,V. et al. (2007) RNAi-based conditional gene knockdown in mice using a U6 promoter driven vector. Int. J. Biol. Sci., 3, 91-99. 
27. Saugspier,M. et al. (2012) Hop bitter acids inhibit tumorigenicity of hepatocellular carcinoma cells in vitro. Oncol. Rep., 28, 1423-1428.

28. Hellerbrand,C. et al. (2005) In situ expression patterns of melanoma inhibitory activity 2 in healthy and diseased livers. Liver Int., 25, 357-366.

29. Stevens,A.P. et al. (2010) Quantification of intermediates of the methionine and polyamine metabolism by liquid chromatography-tandem mass spectrometry in cultured tumor cells and liver biopsies. J. Chromatogr. A, 1217, 3282-3288.

30. Dorn,C. et al. (2010) Xanthohumol, a chalcon derived from hops, inhibits hepatic inflammation and fibrosis. Mol. Nutr. Food Res., 54 (suppl. 2), S205-S213.

31.Zhang,H. et al. (2006) Involvement of programmed cell death 4 in transforming growth factor-beta1-induced apoptosis in human hepatocellular carcinoma. Oncogene, 25, 6101-6112.

32.Zhang,S. et al. (2009) Programmed cell death 4 (PDCD4) suppresses metastastic potential of human hepatocellular carcinoma cells. J. Exp. Clin. Cancer Res., 28, 71.

33. Eferl,R. et al. (2003) Liver tumor development. c-Jun antagonizes the proapoptotic activity of p53. Cell, 112, 181-192.

34. Liu,P. et al. (2002) Activation of NF-kappa B, AP-1 and STAT transcription factors is a frequent and early event in human hepatocellular carcinomas. $J$. Hepatol., 37, 63-71.

35. Desmoulière,A. et al. (2004) The stroma reaction myofibroblast: a key player in the control of tumor cell behavior. Int. J. Dev. Biol., 48, 509-517.

36. Amann,T. et al. (2009) Activated hepatic stellate cells promote tumorigenicity of hepatocellular carcinoma. Cancer Sci., 100, 646-653.

37. Tacchini,L. et al. (2001) Hepatocyte growth factor signalling stimulates hypoxia inducible factor-1 (HIF-1) activity in HepG2 hepatoma cells. Carcinogenesis, 22, 1363-1371.

38. Kim,K.R. et al. (2002) Hypoxia-induced angiogenesis in human hepatocellular carcinoma. J. Mol. Med. (Berl)., 80, 703-714.

39. Rosmorduc, O. et al. (2010) Hypoxia: a link between fibrogenesis, angiogenesis, and carcinogenesis in liver disease. Semin. Liver Dis., 30, 258-270.

40. Warnecke,C. et al. (2004) Differentiating the functional role of hypoxiainducible factor (HIF)-1alpha and HIF-2alpha (EPAS-1) by the use of RNA interference: erythropoietin is a HIF-2alpha target gene in Hep3B and Kelly cells. FASEB J., 18, 1462-1464.

41. Shahrzad,S. et al. (2007) Induction of DNA hypomethylation by tumor hypoxia. Epigenetics, 2, 119-125.
42. Liu,Q. et al. (2011) Hypoxia induces genomic DNA demethylation through the activation of HIF- $1 \alpha$ and transcriptional upregulation of MAT2A in hepatoma cells. Mol. Cancer Ther., 10, 1113-1123.

43. Cai,J. et al. (1996) Changes in S-adenosylmethionine synthetase in human liver cancer: molecular characterization and significance. Hepatology, 24, 1090-1097.

44. Lu,S.C. et al. (2008) S-Adenosylmethionine in cell growth, apoptosis and liver cancer. J. Gastroenterol. Hepatol., 23 (suppl. 1), S73-S77.

45. Huang,G.W. et al. (2005) Expression of hypoxia-inducible factor 1alpha and vascular endothelial growth factor in hepatocellular carcinoma: impact on neovascularization and survival. World J. Gastroenterol., 11, $1705-1708$.

46. Le Bail,B. et al. (1999) Osteonectin/SPARC is overexpressed in human hepatocellular carcinoma. J. Pathol., 189, 46-52.

47. Lee,S. et al. (2012) Differentially expressed genes regulating the progression of ductal carcinoma in situ to invasive breast cancer. Cancer Res., $\mathbf{7 2}$ $4574-4586$

48. Hou,R. et al. (2006) The Fat1 cadherin integrates vascular smooth muscle cell growth and migration signals. J. Cell Biol., 173, 417-429.

49. Moeller,M.J. et al. (2004) Protocadherin FAT1 binds Ena/VASP proteins and is necessary for actin dynamics and cell polarization. EMBO J., $\mathbf{2 3}$, 3769-3779.

50. Sadeqzadeh,E. et al. (2014) Sleeping giants: emerging roles for the fat cadherins in health and disease. Med. Res. Rev., 34, 190-221.

51.Dubuisson,L. et al. (2001) Expression and cellular localization of fibrillin-1 in normal and pathological human liver. J. Hepatol., 34 514-522.

52. Faouzi,S. et al. (1999) Myofibroblasts are responsible for collagen synthesis in the stroma of human hepatocellular carcinoma: an in vivo and in vitro study. J. Hepatol., 30, 275-284.

53. Whittaker,S. et al. (2010) The role of signaling pathways in the development and treatment of hepatocellular carcinoma. Oncogene, 29 , 4989-5005

54. Gomez-Santos,L. et al. (2012) SAMe and HuR in liver physiology: usefulness of stem cells in hepatic differentiation research. Methods Mol. Biol. 826, 133-149.

Received October 25, 2013; revised February 17, 2014;

accepted February 22, 2014 